\title{
Comparing the Dissolution Profiles of Seven Metformin Formulations in Simulated Intestinal Fluid
}

e-mail: raimar@ualberta.ca

\author{
A. Villarroel Stuart ${ }^{1,2}$, Y. Clement ${ }^{1}$, P. Sealy ${ }^{1}$, R. Löbenberg ${ }^{2, *}$ \\ L. Montane-Jaime ${ }^{1}$, R. G. Maharaj ${ }^{1}$, and A. Maxwell ${ }^{1}$ \\ ${ }^{1}$ University of the West Indies, St. Augustine, Trinidad \\ 2 University of Alberta, Alberta, Canada
}

\begin{abstract}
Dissolution testing is used to evaluate the performance of pharmaceutical products. This study compared the dissolution profiles of seven immediate-release metformin $500-\mathrm{mg}$ formulations, including two products with the trade name Glucophage, in simulated intestinal fluid (SIF) at pH 6.8 buffer without enzymes. Other characteristics investigated were absolute weight and drug content. These formulations were marketed in Trinidad and Tobago and are categorized as Class 3 drugs by the Biopharmaceutics Classification System (BCS). The paddle apparatus was used at $75 \mathrm{rpm}$ in $900 \mathrm{~mL}$ of $\mathrm{SIF}$ at $37 \pm 0.5^{\circ} \mathrm{C}$. Samples of $1.25 \mathrm{~mL}$ were removed at 10, 15, 20, 30, 45, and $60 \mathrm{~min}$ without replacement, of which $100 \mu \mathrm{L}$ was diluted with fresh medium and then analyzed spectrophotometrically at $233 \mathrm{~nm}$. Data were analyzed statistically using one-way analysis of variance and DDSolver Add-In to Excel. The weights were significantly different $(p \leq 0.05)$, and each of the formulations demonstrated drug content between $99 \%$ and $103 \%$. Only one of the formulations showed $<85 \%$ drug release in 15 min and a calculated $f_{2}$ value less than 50 , which deviates from the acceptance criteria recommended by the World Health Organization (WHO).
\end{abstract}

KEYWORDS: Metformin; generic drugs; Biopharmaceutics Classification System (BCS); dissolution; Trinidad and Tobago.

\section{INTRODUCTION}

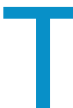
he U.S. FDA has enacted laws to protect patients from substandard pharmaceutical medications (1); these include labeling (1906), safety (1938), effectiveness (1962), and price (1984). The Drug Price Competition and Patent Term Restoration Act (Hatch-Waxman Act) of 1984 and the amendments passed in June 2003 have promoted the entry of generic drugs into the pharmaceutical market (1). Since then, the use of generic medicines has increased. Additionally, other countries have enforced rules to provide safe, effective, and good quality medications for their population (1). Trinidad and Tobago, a member of the World Trade Organization, complies with International Patent laws and embraces the benefits of generic drugs, especially that of accessibility and cost, by including them on the national drug formulary (2). Regardless of these advantages, there are concerns about the safety, efficacy, and quality of generic preparations used in this twin-island republic (3).

Quality control (QC) is described as the most basic step in quality as opposed to quality assurance (QA) and quality management (QM). QC pertains to the finished product and signals defects or deviations from set standards in pharmacopoeias and other bona fide documents $(4,5)$. For pharmaceutical products, there are different aspects of the formulation that must be examined to ensure quality. Various analyses are employed to inspect characteristics such as hardness, friability, and drug content. Performance is tested via disintegration and dissolution (5). The dissolution method has been highlighted as one of the most important techniques because drugs need to be in solution before they are absorbed into the systemic circulation, and the dissolution process measures the rate and extent of drug dissolution (6). Furthermore, medicines with the same active ingredient, strength, and dosage form (pharmaceutically equivalent products) can be compared in vitro to ensure similarity $(7,8)$. Drug regulatory authorities (DRAs) have included these tests among others as prerequisites for the registration and market access of medicines in their countries (7).

Dissolution or "mass transfer" is a procedure that involves the movement of particles from a solid into a solvent (medium) where the particles then go into solution. Dissolution therefore depicts a kinetic process, and its rate signifies the amount of drug that is released over a specified time, which is indicative of its performance (9). The applicability of the dissolution process has expanded since its debut to areas where it replaces in vivo clinical trials for some active ingredients (8). Nevertheless, its initial function as a QC method is frequently used by regulators to confirm the quality and performance for a variety of drugs. In vitro dissolution testing is described in the pharmacopoeias as the release of drug at one or more time points in buffers $(5,9)$.

${ }^{*}$ Corresponding author. 
The World Health Organization (WHO) formulates two lists of pharmaceutical drugs, known as the WHO Model Lists of Essential Medicines (10). These comprise medicines that are used for chronic medical conditions, such as diabetes and hypertension. The purpose of these lists is to provide affordable and accessible medicines for patients worldwide. Metformin and glibenclamide are the only two oral antihyperglycemic drugs on the WHO Model Lists of Essential Medicines. Metformin is indicated for non-insulin dependent diabetes mellitus (NIDDM) or Type 2 diabetes and is the first-line treatment for obese diabetic patients. It is the sole medication in the biguanide class, and unlike the sulfonylureas such as glibenclamide, it is less likely to cause hypoglycemia (11). The absorption of metformin takes place in the intestines, which is approximately $\mathrm{pH}$ 6.8 analogous to simulated intestinal fluid (SIF) media described in the USP (5). The objective of this research was to compare the dissolution profiles in SIF as well as the weights and drug content of seven immediate-release (IR) metformin 500-mg formulations commercially distributed in Trinidad and Tobago, of which five were generic formulations and the other two products had the same trade name as the innovator, Glucophage, but came from different manufacturers.

\section{MATERIALS AND METHODS Reagents}

Potassium phosphate monobasic and sodium hydroxide were purchased from Caledon Laboratories Limited. Hydrochloric acid NF/FCC grade was obtained from Fisher Scientific.

\section{Tablet Characteristics}

The seven IR metformin 500-mg tablets, two of which were Glucophage-branded products of different manufacturers, were bought from drug distributors in Trinidad, and the metformin standard was purchased from Sigma-Aldrich. Twenty tablets of each formulation were weighed individually, and the weight variation was calculated using Microsoft Excel 2007. The drug content was determined by the USP method (5) using a Milton Roy Spectronic 3000 Array Spectrophotometer with RapidScan software and Hellma quartz glass SUPRASIL 200-2500 nm cuvettes. Six samples were taken from twenty crushed tablets of each formulation, and the mean absorbance and concentration were obtained and used to calculate the percentage of metformin in each product. A calibration curve with an $R^{2}$ value of 0.999 was applied to compute the concentration calculations.

\section{Dissolution}

SIF medium without enzyme was prepared according to the USP: Dissolve $6.8 \mathrm{~g}$ of monobasic potassium phosphate in $250 \mathrm{~mL}$ of water, mix, and add $77 \mathrm{~mL}$ of $0.2 \mathrm{~N}$ sodium hydroxide and $500 \mathrm{~mL}$ of water, adjust the resulting solution with either $0.2 \mathrm{~N}$ sodium hydroxide or $0.2 \mathrm{~N}$ hydrochloric acid to a pH of $6.8 \pm 0.1$. Dilute with water to $1000 \mathrm{~mL}$.

Deaeration of the SIF medium was done by applying vacuum and filtering through a $0.45-\mu \mathrm{m}$ membrane filter while sonicating with a Branson Model 8200 Bransonic Ultrasonic Cleaner water bath. The phosphate medium was then preheated, weighed, and placed in a water bath of a six-vessel Model VK 7020 VanKel Dissolution Tester, Apparatus 2 (paddle), at $75 \mathrm{rpm}$, with a Model VK 8000 Varian System Monitor Sampling Station. Each tablet was weighed and subsequently placed in one of the six vessels that contained $900 \mathrm{~mL}$ of deaerated SIF medium. Samples of $1.25 \mathrm{~mL}$ were withdrawn through $70-\mu \mathrm{m}$ Varian filters at $10,15,20,30,45$, and 60 min without medium replacement; subsequently $100 \mu \mathrm{L}$ of this sample was placed in a culture tube containing $4.9 \mathrm{~mL}$ of fresh medium. These samples were covered with foil paper until analysis with a UV spectrophotometer at $233 \mathrm{~nm}$, and fresh medium was used as the blank. A calibration curve was used for the quantification of the drug.

\section{Statistical Analyses}

Minitab version 16; Statistical Package for the Social Sciences (SPSS) for Windows versions 12; Microsoft Office Excel 2007; and DDSolver software, an Add-In to Excel 2007, were used for the statistical analysis (12). The latter was used to calculate the similarity $\left(f_{2}\right)$ and difference $\left(f_{1}\right)$ factors, dissolution efficiency $(D E)$, mean dissolution time $(M D T)$, and area under the release curve $(A \cup C)$. The similarity factor $f_{2}$ was calculated only if either the reference or test product released less than $85 \%$ of the active ingredient in 15 min with $n=12$; all other dissolution tests were $n=6$. Dunnett's method compared the control formulation (Innovator 1) with the other formulations and gave $p$ values. The Ryan-Einot-Gabriel-Welsch (REGW) F-test is a multiple step-down range post-hoc test that produced homogeneous subsets for the dissolution means of the seven formulations. This was performed to determine whether the different products were statistically similar.

\section{RESULTS}

Innovator 1 and 2 had similar average weights of $0.531 \pm 0.006 \mathrm{~g}$, and the same was true for Formulations 4 and 5 with values of $0.588 \pm 0.010 \mathrm{~g}$ and $0.584 \pm 0.008$ $\mathrm{g}$, respectively. However, Formulation 2, with an average weight of $0.649 \pm 0.007 \mathrm{~g}$, had the highest weight of the seven formulations followed by Formulation 3 with $0.618 \pm 0.010 \mathrm{~g}$ (Table 1). All of the products except Formulation 2 released $\geq 85 \%$ of metformin drug in $15 \mathrm{~min}$ in pH 6.8 SIF buffer, and the dissolution profiles demonstrate that Innovator 1 released the highest amount of metformin followed closely by Formulation 1 (Figure 1). The ANOVA table for the dissolution profiles of the seven formulations gave a $p$ value of 0.002 . Dunnett's method gave a $p$ value greater than 0.05 for Formulation 1 (0.091) as opposed to the $p$ values for the other products, which were less than 
Table 1. Characteristics of Metformin 500-mg Formulations

\begin{tabular}{lclllll}
\hline & Average weight (g) & $(\mathrm{SD} \pm)$ & Assay (\%) & Country & Manufacturer & Lot Number \\
Innovator 1 & 0.531 & 0.006 & 102.5 & France & Lipha Sante & 250478 \\
Innovator 2 & 0.531 & 0.006 & 103.3 & Spain & Merck Serono & 11678 \\
Formulation 1 & 0.575 & 0.009 & 98.8 & India & Aurochem & MF52 \\
Formulation 2 & 0.649 & 0.007 & 103.1 & Germany & Denk Pharma & $8 \mathrm{~B} 7$ \\
Formulation 3 & 0.618 & 0.010 & 101.6 & India & Intas & VL1195 \\
Formulation 4 & 0.588 & 0.010 & 102.5 & Canada & Apotex & $(\mathrm{L})$ JR0691 \\
Formulation 5 & 0.584 & 0.007 & 101.9 & India & Cipla & DG0597 \\
Average weights showed $a p \leq 0.05$. & & & & & & \\
SD=Standard Deviation; $n=20$ & & & & & & \\
\hline
\end{tabular}

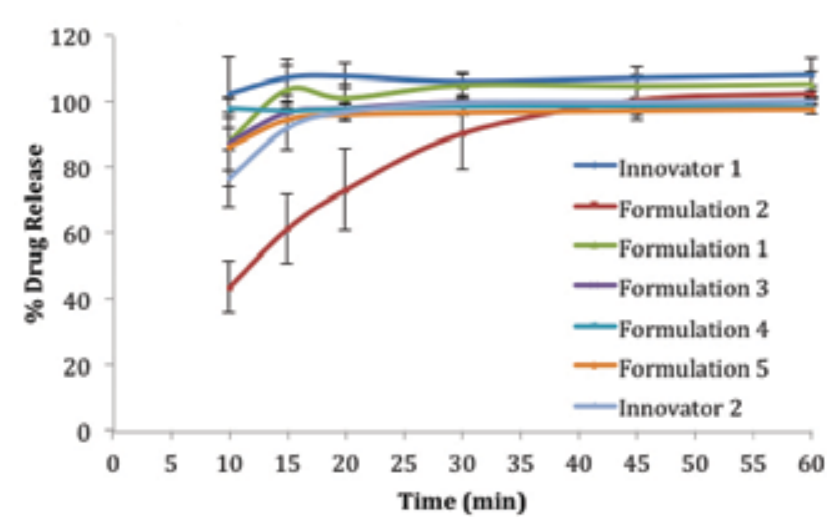

Figure 1. Dissolution profiles of metformin formulations in SIF.

and Formulation 2 were the only formulations in subsets 1 and 4 , respectively.

All the formulations contained approximately $100 \%$ metformin active ingredient in purified water, but Innovator 2 and Formulation 2 produced higher values of $103.3 \%$ and $103.1 \%$, respectively, as opposed to Formulation 1, which had the lowest percentage of metformin (98.8\%). However, formulation behavior in SIF depicted Innovator 1 with the largest value for the AUC (5855.9 $\mathrm{min} \%$ ) over the $60-\mathrm{min}$ period followed by Formulation 1 (5595.3 min\%), while Formulation 2 exhibited the lowest AUC (4582.7 $\mathrm{min} \%)$. Formulation 4 (5.2 $\mathrm{min}$ ) and Innovator 1 (5.8 $\mathrm{min}$ ) had the lowest $M D T$, and Formulation 2 displayed the highest $M D T$ (15.3 $\mathrm{min}$ ) out of the seven products. Innovator $1(0.98 \%)$, Formulation $1(0.9 \%)$, and Formulation $4(0.9 \%)$, in that order, showed the highest $D E$, whereas Formulation 2 $(0.7 \%)$ showed the lowest value (Table 2 ).

Table 2. Parameters for Characterizing Drug Release Curve for Metformin 500-mg Formulations in pH 6.8 Phosphate Buffer

\begin{tabular}{lccc}
\hline Parameters & AUC $(\min \%)$ & $M D T(\min )$ & $D E(\%)$ \\
Innovator 1 & 5855.9 & 5.8 & 0.976 \\
Innovator 2 & 5242.2 & 7.5 & 0.874 \\
Formulation 1 & 5595.2 & 6.8 & 0.933 \\
Formulation 2 & 4582.7 & 15.3 & 0.764 \\
Formulation 3 & 5358.1 & 6.5 & 0.893 \\
Formulation 4 & 5390.1 & 5.2 & 0.898 \\
Formulation 5 & 5236.3 & 6.3 & 0.873
\end{tabular}

$A U C=$ Area under the release curve; $M D T=$ Mean Dissolution Time; $D E=$ Dissolution Efficiency
The $f_{2}$ values of 18.9 and 29.5 and $f_{1}$ values of 26.3 and 17.6 were obtained for Innovator 1 and 2, respectively, when compared with Formulation 2 (Table 3).

Table 3. Summary of Similarity, $f_{2}$, and Difference Factors, $f_{1}$, for Formulation 2 Compared with Innovators 1 and 2

\begin{tabular}{ccc}
\hline$p H 6.8$ & Formulation 2 $\left(f_{2}\right)$ & Formulation 2 $\left(f_{1}\right)$ \\
\hline Innovator 1 & 18.9 & 26.3 \\
\hline Innovator 2 & 29.5 & 17.6 \\
\hline
\end{tabular}

$\geq 50$ and $<15$ represent similarity via $f_{2}$ and $f_{1}$ calculations, respectively.

\section{DISCUSSION}

The use of $f_{2}$ to determine similarity between and among the dissolution profiles of drug products is recommended by the FDA, the European Medicines Agency, and WHO; hence, it was applied to the release profiles in this study (13). Furthermore, a multiple-point dissolution investigation was used rather than single-point comparisons for the seven metformin preparations.

Metformin is soluble in water, slightly soluble in alcohol, and insoluble in substances such as acetone, ether, and chloroform (14). This study analyzed metformin dissolution properties in $\mathrm{pH} 6.8$ buffer by measuring its release, which was $\geq 85 \%$ in 15 min for most of the formulations investigated. However, Formulation 2 released $\geq 85 \%$ of metformin in $15 \mathrm{~min}$ and had an $f_{2}$ value that was less than 50 when compared with the Innovator products, thus indicating a performance difference from the comparator products in phosphate buffer. Furthermore, Formulation 2 had the longest dissolution time and the lowest area under the dissolution curve. Sheorey et al. (15) and Zakeri-Milani et al. (16) have shown similar findings to our study, as they reported that one of the metformin formulations examined in their studies did not satisfy the requirements of the pharmacopeia, that is, $75 \%$ or $80 \%$ drug release in 45 or $30 \mathrm{~min}$, respectively, depending on whether the basket $(100 \mathrm{rpm})$ or the paddle $(100 \mathrm{rpm})$ was used in that order.

The WHO requirement for drugs in Class 3 of the BCS, such as metformin, is very rapid dissolution; specifically, they must release $85 \%$ or more of the drug in $15 \mathrm{~min}$ at 
$75 \mathrm{rpm}$ using multiple time points (7). This differs from the USP (5), which specifies an $80 \%$ release in 30 min (single time point) using the paddle at $50 \mathrm{rpm}$. This study used $75 \mathrm{rpm}$ because coning can occur at a speed of $50 \mathrm{rpm}$ and can affect the dissolution results (17). It is evident therefore that the standard set by WHO is more rigid than that of the USP; the former can be used to establish in vitro equivalence between an innovator and generic drug product but requires three distinct media of different $\mathrm{pH}$ values $(7,9)$. Although our investigation looked at the in vitro dissolution of metformin in a single buffer, which is the mainstay in pharmacopoeias, the WHO standard (i.e., $\geq 85 \%$ in $15 \mathrm{~min}$ ) should be considered (7).

The observed dissolution profile of Formulation 2 can be attributed to the excipients, the manufacturing process, or both, used for this particular product (18).

Nonetheless, the formulations investigated were all within the weight range of $\pm 5 \%$ deviation for tablets with an average weight greater than $250 \mathrm{mg}$, and their drug contents were similar.

L.C. Block et al. (18) performed a study in which different excipients were tested in metformin tablets manufactured via wet granulation and determined that the amount and type of excipients used in the metformin products modified their dissolution behavior.

In our study, there were two products with the same trade name. These two products both released $\geq 85 \%$ metformin drug in $15 \mathrm{~min}$; however, the profile for Innovator 1 is closer to that of Formulation 1 than that of Innovator 2. The Dunnett method also verified this finding statistically as Formulation 1 with a $p$ value greater than 0.05 was the only product similar to Innovator 1 . To select a reference product as the comparator for generic preparations, the innovator is commonly chosen as the reference. However, in this case, there were two products called Glucophage that were both from Europe (France and Spain), where they were first introduced. Subsequently, Bristol Myers Squibb was the manufacturer for this drug in the United States (19). However, two products that carry the same name can be assumed to be identical. This is similar to results reported by Löbenberg et al. (20) for metronidazole products with an identical name. It is also imperative for generic preparations to show similarity to the innovator with respect to safety, efficacy, and quality. The generic drug is only as good as its comparator; hence, selecting a reference drug for comparison to generic products is crucial (7). The WHO guideline for selecting a comparator or reference product is ranked as follows:

1. Choose the innovator for the product under investigation at a national level.

2. Use the WHO reference list for a comparator.

3. Use the International Conference on Harmonisation (ICH) list for an innovator.

4. If none of the above is available, a suitable comparator (including a generic) that has proved to be similar in safety, quality, and efficacy to the innovator can be used.

\section{CONCLUSION}

This study examined the quality parameters of seven immediate-release metformin $500 \mathrm{mg}$ tablets including dissolution in SIF, weight variation, and drug content and found six metformin formulations that fulfilled the WHO requirements in regard to in vitro dissolution behavior. The two innovator products with the same trade name were statistically different but had similar biopharmaceutical qualities. This demonstrates that the same trade name does not necessarily indicate that products are identical, especially if they have different manufacturers. Government organizations and patients have benefited from generic drugs because of their cost-effectiveness; however, their quality, incorporating dissolution behavior, must be investigated relative to a suitable comparator.

\section{REFERENCES}

1. Young, A. R. Generic Pharmaceutical Regulation in the United States with Comparison to Europe: Innovation and Competition. Wash. U. Global Stud. Law Rev. 2009, 8 (1), 165-185. http://openscholarship.wustl.edu/ cgi/viewcontent.cgi?article $=1071 \&$ context $=$ law $_{-}$ globalstudies (assessed Jan 12, 2015).

2. Trinidad and Tobago: Pharmaceutical Country Profile, 2012. The Ministry of Health of Trinidad and Tobago and Pan American Health Organization. World Health Organization Web site. http://www.who.int/ medicines/areas/coordination/PSCP_TRT_en.pdf (assessed Jan 12, 2015).

3. Pereira, L. M. P.; Fraser, H.; Burnett, F. Assessment of Generic Drugs in the Caribbean. Drug Inf. J. 1998, 32 (1), 145-150. DOI: $10.1177 / 009286159803200120$.

4. The International Pharmacopeia, 3rd ed.; World Health Organization: Geneva, 1994; Vol. 4.

5. The United States Pharmacopeia and National Formulary USP 35-NF 30; The United States Pharmacopeial Convention, Inc.: Rockville, MD, 2012.

6. Azarmi, S., Roa, W. H., Löbenberg, R. Targeted delivery of nanoparticles for the treatment of lung diseases. Adv. Drug Del. Rev. 2008, 60 (8), 863-875. DOI: 10.1016/j. addr.2007.11.006.

7. WHO Expert Committee on Specifications for Pharmaceutical Preparations. Multisource (generic) pharmaceutical products: guidelines on registration requirements to establish interchangeability; WHO Technical Report Series, No. 937, Annex 7; World Health Organization: Geneva, 2006; pp 347-438. http://apps. who.int/prequal/info_general/documents/TRS937/ WHO_TRS_937_eng.pdf\#page=359 (assessed Jan 12, 2015).

8. Waiver of In Vivo Bioavailability and Bioequivalence Studies for Immediate-Release Solid Oral Dosage Forms Based on a Biopharmaceutics Classification System; Guidance for Industry; U.S. Department of Health and Human Services, Food and Drug Administration, Center for Drug Evaluation and Research (CDER), U.S. Government Printing Office: Washington, DC, 2000. 
http://www.fda.gov/downloads/Drugs/Guidances/ ucm070246.pdf (accessed Jan 12, 2015).

9. Krämer, J.; Grady, L. T. Gajendran, J. Historical Development of Dissolution Testing. In Pharmaceutical Dissolution Testing; Dressman, J., Krämer, J., Eds.; Taylor \& Francis Group: Boca Raton, FL, 2005; 1-37. DOI: 10.1201/9780849359170.ch1.

10. WHO Model List of Essential Medicines 18th ed. (April 2013). World Health Organization Web site. http://www. who.int/medicines/publications/essentialmedicines/ en/ (accessed Jan 12, 2015).

11. Katzung, B. G.; Masters, S. B.; Trevor, A. J. Basic and Clinical Pharmacology, 11th ed.; McGraw-Hill: New York, 2009.

12. Zhang, Y.; Huo, M.; Zhou, J.; Zou, A.; Li, W.; Yao, C.; Xie, S. DDSolver: An Add-In Program for Modeling and Comparison of Drug Dissolution Profiles. AAPS J. 2010, 12 (3), 263-271. DOI: 10.1208/s12248-0109185-1. http://www.ncbi.nlm.nih.gov/pmc/articles/ PMC2895453/ (assessed Jan 12, 2015).

13. Costa, P.; Sousa Lobo, J. M. Modeling and comparison of dissolution profiles. Eur. J. Pharm. Sci. 2001, 13 (2), 123-133. DOI: 10.1016/S0928-0987(01)00095-1.

14. The Merck Index, 15th ed.;O'Neil, M. J., Ed.; The Royal Society of Chemistry: London, 2013.

15. Sheorey, S. D.; Hinge, M. A.; Sengupta, R.; Menon, B. V. Pharmaceutical Equivalence Between Different Brands of Metformin Hydrochloride Tablets. J. Pharm. Res. 2012, 5 (6). http://www.jpronline.info/index.php/jpr/ article/download/13880/7047 (assesed Jan 12, 2015).

16. Zakeri-Milani, P.; Nayyeri-Maleki, P.; Ghanbarzadeh,
S.; Nemati, M.; Valizadeh, H. In-vitro bioequivalence study of 8 brands of metformin tablets in Iran market. J. Appl. Pharm. Sci. 2012, 2 (8), 194-197. DOI: 10.7324/ JAPS.2012.2834.

17. Higuchi, M.; Yoshihashi, Y.; Tarada, K.; Sugano, K. Minimum rotation speed to prevent coning phenomena in compendium paddle dissolution apparatus. Eur. J. Pharm. Sci. 2014, 65 (18), 74-78. DOI: 10.1016/j.ejps.2014.09.010.

18. Block, L. C.; Schemling, L. O.; Couto, A. G.; Mourão, S. C.; Bresolin, T. M. B. Pharmaceutical equivalence of metformin tablets with various binders. Rev. Cienc. Farm. Basica Apl. 2008, 29 (1), 29-35. http://www.servbib.fcfar.unesp.br/seer/index.php/Cien_Farm/article/ viewFile/431/414 (assessed Jan 12, 2015).

19. Orange Book: Approved Drug Products with Therapeutic Equivalence Evaluations. U.S. Food and Drug Administration Web site. http://www.accessdata. fda.gov/scripts/cder/ob/docs/tempai.cfm (assessed Jan 12, 2015).

20. Löbenberg, R.; Chacra, N. B.; Stippler, E. S.; Shah, V. P.; DeStefano, A. J.; Hauck, W. W.; and Williams, R. L. Toward Global Standards for Comparator Pharmaceutical Products: Case Studies of Amoxicillin, Metronidazole, and Zidovudine in the Americas. AAPS J. 2012, 14 (3), 462-472. DOI: 10.1208/s12248-012-9350-9. 